\title{
Les institutions de l'Union Europeenne : le status, les competences et l'equilibre institutionnel en face de l'elargissement
}

Ana Djoric

\section{Bref apperçu historique de l'integraton européenne}

L'idée d'unification européenne a des noumbreux participatns et elle n'est pas nouvelle. On peut la trouver à l'Est aussi bien qu'à l'Ouest, au Nord et au Sud du continent, chez les philosophes, juristes ou écrivains. Mais les différences dans l'approche empechait sa mise en place. Les inégalités causaient des antagonismes qui menait les peuples en guerres sanglants, qui devastaient le continent européen. Cela posait un problème grave sur la route d'unification. Maintenir la paix en Europe était la première chose à faire, pour réaliser la conception des Etats-Unis d'Europe.

La sauvegarde de la paix, une fois atteint, menait vers l'union economique au profit de tous les citoyens vivant en Europe, car l'expériece de la chute d'intégration politique était déjà acquise'. L'unification économique ${ }^{2}$ a toujours été le moteur du processus d'unification européenne. Il fallait, donc, établir un marché commun. Une fois l'integration economique réalisée, les peuples européens

'Le Projet Briand, présenté par le ministre français en 1929 à l'Assemblée générale de la Société des Nations parlait d'une organisation fédérale.

${ }^{2}$ Robert Schuman, ministre des affaires étrangères de France, et un des pères d'Europe unie, dans son plan Shuman: "L'Europe ne se fera pas d'un coup, ni dans une construction d'ensemble; elle se fera par des réalisations concrètes créant d'abord une solidarité de fait ". Le fonctionnalisme écarte ainsi à la fois l'intégration globale au profit d'une intégration par secteurs et l'intégration politique au profit de l'intégration économique. 
peuvent se précipiter à la recherche de l'unité politique, ainsi que vers le renforcement de la cohésion sociale.

Dans l'unification européenne, on peut distinguer la coopération ${ }^{3}$, et l'intégration. Dans le modèle de coopération il n'est pas prévu la création d'un Etat central, mais seulement d'unir les pays souverains en une confédération dans laquelle les structures nationales demeurent inchangées. L'intégration va au délà de la coéxistence traditionnelle d'Etats nationaux. La conception classique de l'inviolabilité et de l'indivisibilité de la souveraineté des Etats fait place à la conviction qu'il ne peut pas être remédié aux imperfections des relations entre l'individu de l'Etat, aux insuffisances des systèmes nationaux et aux abus de pouvoir d'un Etat vis-à-vis d'autres, que si les différentes souverainetés nationales sont unies en une souveraineté commune et fusionnent à un niveau supérieur dans une communauté supranationale. Ce processus doit aboutir à la création d'un Etat fédéral européen dans lequel les individus remettent leur destinée entre les mains d'autorités communautaires, mais qui préserve les particularités des nations constituant cet Etat (fédération) 4 .

Pour un observateur non averti de l'Europe, l'unification européenne a une image confuse à cause de la multiplicité et la compléxité des différentes organisations.

En Europe aujourd'hui coéxistent, sans réel lien, l'Organisaton de coopération et de développement économique $(\mathrm{OCDE})^{5}$, L'Union de l'Europe occidentale (UEO) ${ }^{6}$, L'Union du traité de l'Atlantique Nord $(\mathrm{OTAN})^{7}$, le Conseil de l'Europe ${ }^{8}$ et l'Union européenne.

${ }^{3}$ Le Conseil d'Europe, l'OCDE, ainsi que le deuxième et le troisième piliers de l'UE sont faits sur ce concept.

${ }^{4}$ L'UE est fondée sur ce concept.

'A l'origine de l'OCDE est l'Organisaton Européenne de Coopération économique - OECE fondée le 16 avril 1948, crée pour la répartition de l'aide américdaine du plan Marshall ; en 1961 l'OCDE se substitue à l'OECE; Elle est à la fois un lieu de rencontre et de négociation au sujet des échanges économiques, dans le sens de la croissance et du libre échange, et une instance de réfexion et d'analyse sur les économies nationales et transnationales:

"Les accords de Paris, signés le 23 octobre 1954, entrés en vigueur le 1 mai 1955 renforcent l'OTAN en instituent l'UEO. Depuis l'initiative française pour la réactivation de l'UEO en 1984 (Déclaration de Rome), le rôle de l'UEO s'agrandit. Le Traité de Maastricht lui confere mission actif de maintien de la sécurité.

Les Européens de l'Ouest et leurs alliés américains établissent par le Traité de 
L'Union européenne (UE) a ćté crée par le traité de Maastricht en $1992^{9}$. Le traité sur l'UE comporte trois "piliers". Le premier pilier englobe trois communautés européennes, la Communauté européenne ou, à l'origine, la Communauté Economique européenne (CEE), qui a une compétence économique et générale, la Communaute européenne pour l'Energie atomique (CEEA ou EURATOM) et la Communauté européenne pour le Charbon et Acier (CECA) qui sont spécialisées. Le deuxième pilier est la politique étrangère et de sécurité commune (PESC). Troisième pilier est la coopération dans les domaines de la justice et des affaires intérieures (JAI). Le deuxième et le troisième pillier presentent la coopération interétatique entre les Etats membres et procèdent d'accords adoptés à l'unanimité.

Les Communautés curopéennes peuvent être caractérisées par trois traits fondamentaux. Tout d'abord, les communautés européennes sont, suivant les règles du droit internaitonal, les organisations internationales. Elles sont fondées par les traités fondateurs. En même temps, il s'agit des organisations régionales ouvertes, de type spécialisé. La personnalité morale est expressément conférée à chacune des

l'Atlantique Nord le 4 avril 1949 une organisation de légitime défense, conforme à la Charte des Nations Unies. L'article 5 du traité engage les Etats membres à aider l'un d'entre eux qui serait attaqué. Progressivement le traité a été concrétisé par des structures militaires et une véritable organisation s'est mise en place. Après la dissolution du Pacte de Varsovie, l'OTAN est maintenue, malgré les changements à l'est de l'Europe. Certains Etats d'Europe centrale souhaite y être admis, mais la Russie s'y oppose aussi l'OTAN a proposé un "partenariat pour la paix " en janvier 1994. L'adhésion au Partenariat implique le respect de principes essentiels, la démocratie, le règlement négocié des conflits et le contrôle civil du militaire.

"Le 19 septembre 1946 à l'Unifersité de Zurich, Winston Churchill lance un appel à la réconciliation franco-allemande et aux Etats Unis d'Europe. Du 7 au 11 mai 1948 se tient le Congrès de la Haye où est arrêtée l'idée d'une Europe par étape et l'adoption d'une Charte des droits de l'Homme. Le Conseil de l'Europe apparaît comme l'organisation de l'Europe des démocraties; l'admission au Conseil de l'Europe apparaît comme un rattachement à l'Europe de l'ouest et un passage obligé avant d'adhérer à la CEE. Pour devenir membre du Conseil de l'Europe, il faut respecter les droits de l'homme et la démocratie. Certains pays d'Europe centrale ne furent admis qu'après avoir adopté des constitutions conformes, respectant les libertés indifviduelles et collectives, la démocratie et le pluripartisme.

"Selon l'article A : "sur les communautés européennes complétées par les politiques et formes de coopération instaurées". 
Communautés par le traité ${ }^{10}$. La spécialité des Communautés est déterminée de deux manières : d'abord dans son domaine pour ces Communautés sectorielles que sont la CECA et la CEEA ; ensuite par sa nature qui, pour les trois Communautés, en fait des organisations économiques par référence à la notion de "marché commun ". La troisième caractéristique des Communautés est qu'elles sont fondées sur un mode particulier d'intégration économique, la constitution d'un "marché commun" qui, par ses implications est susceptible d'aller très au-delà de cette seule intégration. La CEE est rebaptisé dans le traité instituant l'UE, car elle abandonne l'approche sectorielle des deux autres comunautés, car son objectif est de réunir en une seule Communauté les Etats membres dans tous les domaines de l'économie. Cela s'applique à des domaines aussi essentiels que la libre circulation des marchandises, des travailleurs, des services et des capitaux ainsi que pour la liberté d'établissement et des opérations de paiement, la politique de concurrence, la politique économique et monétaire, la politique agricole, la politique des transports, la politique de l'environnement, la politique en matière de recherche et de technologie ou la politique industrielle.

Le sujet de nos interets sera le pilier communautaire : les institutions et leurs perspectives en vue d'elargissement envisagé pour un avenir proche $^{11}$.

\section{Les Droit Institutionnel de l'Union}

Le Droit institutionnel constitue une partie du Droit Communautaire $^{12}$. Les dispositions du droit institutionnel régissent la constitution des Communautés, leur fonctionnnement et leur contrôle ainsi que l'ordonnancement juridique dont elles sont la source et le support. Donc, le droit institutionnel communautaire regroupe: droit

"Art. 6 CECA, Art. 210 CE, Art. 184 CEEA

"L'UE a commencé le processus de négociations en vue d'élargissement avec 13 pays dont 10 devraient devenir les membres de l'Union en 2004.

${ }^{12}$ Le droit matériel et le droit institutionnel sont les parties composantes du droit communautaire - Boulois, Jean, Droit institutionnel de l'Union européenne, Paris, Montchrestien, 1997 ; Isaak G, Droit communautaire général, Paris, Armand Colin, 1996 
constitutionnel, administratif, budgétaire, international, ou ce qu'on appelle d'habitude le droit public, d'après la conception scolastique française.

\section{La structure institutionnelle}

Dans l'UE on peut faire la distinction entre les institutions et les organes ${ }^{13}$.

Le tratié CE établit une distinction entre les institutions et les organes. Les institutions de la CE sont énumérées à l'article 7 (ex 4). Par rapport aux organes, les institutions disposent d'une moindre autonomie, ce qui s'exprime par exemple dans la désignation de leurs membres ou dans le pouvoir dont elles disposent pour fixer leur propre règlement intérieur. Cette distinction opérée entre les institutions et les autres organes n'a, en pratique, qu'une importance très limitée ${ }^{14}$.

Dans sa version originaire, la structure institutionnelle des Communautés, repose sur quatre institutions : une assemblée, un conseil, une commission et une cour de justice. Les institutions communautaires sont essentiellement chargées de remplir le cadre d'intégration défini par les Etats membres. Au départ, l'accent était mis sur le Conseil et la Commission européenne, lorsque le Parlement européen ${ }^{15}$ a eu seulement un rôle consultatif.

Par le Traité de fusion, signé le 8 avril 1965 et entré en vigueur le $1^{\text {cr }}$ juillet 1967, ont été institués une Commission unique et un Conseil des ministres uniques pour l'ensemble des trois Communautés. Les institutions ne sont plus quatre mais cinq, depuis le traité de Maastricht ${ }^{16}$. La constitution des certaines d'entre elles a été plus ou moins modifiée, altérant ainsi ce qui était leur légitimité fondamentale.

${ }^{13}$ Certains auteurs, comme par exemple J. Boulois font la distinction entre les organes principaux et ceux qui sont subsidiaires, dont l'existence ne se présente pas juridiquement de la même manière, suivant qu'ils ont été ou non prévus par les traités. Or, il s'agit d'une distinction terminologique, sans valeur juridique. (J. Boulois, Droit institutionnel de l'Union européenne, Paris, Montchrestien, 1997)

14 Zbinden Martin, Les institutions et les procédures de prise de décision de l'Unione européenne après Amsterdam, Berne, Staempfli Editions SA, 2002, p. 13 et s.

${ }^{15}$ Le triangle institutionncl.

Ir Le traité de Maastricht introduit la Cour des comptes comme la cinquième institution 
La question de repartition des cometences dans le cadre de l'UE est resoulue sur deux axes, dite horizontale et verticale ${ }^{17}$.

La répartition des cometences entre les institutions européennes est soumise à deux principes. Le premier consiste dans ce que la $\mathrm{CJCE}$ appelle " l'équilibre institutionnel " qui est devenu un principe général de droit communautaire. Le second est formé d'un ensemble de règles qui ont dû être dégagées à la suite des modifications opérées dans cet équilibre. Ces règles concernent le choix de la base juridique des actes en tant qu'il est de nature à influer su r la répartition des fonctions entre les organes.

L' »équilibre des pouvoirs " a été utilisée par la $\mathrm{CJCE}^{18}$. Il s'agit du principe qui prévoit que le rapport des pouvoirs respectivement attribués à la Haute Autorité et aux autres institutions de la Communauté. La répartition des attributions caractéristique du système institutionnel communautaire, n'est pas en effet une modalité d'une telle séparation et ne saurait le devenir pour cette raison que les institutions ne procèdent pas d'une même légitimité.

Les actes des institutions doivent être fondés sur des articles des traités qui constituent leur base légale; celle-ci ne conditionne pas

${ }^{17}$ La réparition des compétences entre l'Union et ses Etats membres doit déterminer les domaines et les matières dans lesquels la première est compétente pour décider et pour agir et ses organes habilités à exercer les pouvoirs dont les investissent les traités. Les principes qui informent la répartition des compétences entre l'Union et ses Etats membres sont énoncés par l'article $3 \mathrm{~B}$, qui cite trois principes. Ce sont le principe de spécialité, le principe de subsidiarité et le principe de proportionnalité. La spécialité est inhérent à la personnalité morale, la personnalité juridique n'étant conféré aux personnes morales que comme instrument de réalisation des objectifs en vue desquels elles ont été crées. Il joue comme une justification des actes et comme une limite au delà de laquelle l'aciton juridique dépourvue de fondement sea frappé de nullité. Le principe de subsidiarite est simple. Il prévoit que la comunauté et l'Union doivent agir si et dans la mesure où les objectifs de l'action envisagée ne peuvent pas être atteints de manière suffisante par une action des Etats membres, partant être mieux réalisés par une action de la Communauté (ou de l'Union). Il concerne seulement les compétences dite "partagées ". La difficulté tient alors à ce qu'il n'existe pas de compétence " partagée " ou " mixte " mais des domaines communs et des objectifs communautaires. En plus, la CJCE a décide que ce principe ne saurait être considéré comme ayant un effet direct. Le principe de proportionnalité determine que l'action de la Communaute n'excède pas ce qui est nécessaire pour atteindre les objectifs du traité. Dans le pratique de la CJCE il a pris valeur de principe général.

${ }^{18}$ Arrêt Meroni, 13/06/1958, n0 9/56, p. 11 
seulement la nature de l'acte parmi ceux que peuvent prendre les institutions. Elle conditionne aussi son contenu et sa procédure d'édiction. L'hypothèse d'une absence de base légale spécifique est réglée par les articles des traités. La procédure applicable se caractérise notament par le fait que le Conseil doit statuer à l'unanimité et que, suivant les dispositions des tratiés CE et CEEA, le Parlement européen n'est que consulté.

\section{Les institutions legislatives}

\subsection{Le Parlement Européen}

Le Parlement Européen (PE) ${ }^{19}$ est composé de représentants des peuples des Etats réunis dans les Communautés.

C'était prévu que ces représentants devraient être élus au suffrage universel direct suivant une procédure uniforme dans les Etats membres. Cependant, jusqu'au 1979 les membres du PE étaient des membres délégués des parlements nationaux. Mais depuis cette annéelà et les premières éléctions au suffrage universel direct, le Parlement est l'organe d'expression démocratique au sens propre du terme.

La constitution du Parlement est réglée par trois séries de dispositions dont les unes concernent sa composition, les autres l'élection et les dernières le status de ses membres.

Le mandat des députés est incompatible avec la qualité du membre du gouvernement d'un Etat membre. Or, il est compatible avec le mandat parlementaire national.

L'organisation et le fonctionnement du Parlement sont régis par des dispositions qui arrêtées pour la plupart dans son règlement intérieur, reproduisent les solutions habituelles du droit parlementaire.

Le Parlement a son siège à Strasbourg, où se tiennent les douze périodes de sessions plénières mensuelles, y compris la session budgétaire. Les périodes de sessions plénières additionnelles se tiennent

19 Lors de la création de la CECA l'Assemble commune a été instituée organe parlamentaire. Dès la création de la CEE et de la CEEA ses compeétenes ont été élargies à ces nouvelles Communautés. En 1962 elle s'est donnée le nom de Parlement européen. Toutefois, cette appellation n'est entrée dans les traités fondateurs que par l'AUE. 
à Bruxelles. Les commissions du Parlement européen siègent à Bruxelles. Le secrétariat général du Parlement européen et ses services restent installés à Luxembourg.

Dans la procédure législative, le rôle de Parlement évolue. Les traités ont considérablement changé le rôle et les competences du Parlement. Son influence dans le processus législatif augmente surtout après le traité sur l'UE qui modifie sensiblement sa situation. Ses pouvoirs ont été étendus progressivement. Au départ, il était prévu que le Parlement doit être consulté. Sa décision ne pouvait pas influencer le travail du Conseil, car son avis n'obligeait pas. Mais, la situation change. En 1975 il a été introduite la procédure de concertation, qui concédait au PE de véritables droits de participation. La conséquence : le PE peut être saisi pour les projets d'actes législatifs qui ont des conséquences notables pour le budget communautaire. Depuis 1975, le PE jouit de pouvoirs importants et de véritables droits de codécision en matière de droit budgétaire.

Depuis l'entrée en vigueur de l'Acte Unique Européen (AU), les accords d'adhésion et d'association avec d'autres Etats nécessitent l'approbation du PE. Une réglementation différenciée régit les autres accords internationaux.

L'Acte unique, puis le traité sur l'UE introduisent d'abord un avis conforme sur certain nombre de cas; en plus, il commence la mise en place de procédures de coopération. Il prend de plus en plus de compétences. La consultation du Parlement marque le changement dans la répartition des pouvoirs. Avec le traité sur l'Union, entré en vigueur fin 1993, la procédure législative la plus importante devient la procédure de codécision nouvellement introduite. Elle régit un bon nombre de domaines majeurs du pilier communautaire, tels le marché intérieur, la culture, l'éducation, la santé, la recherche et l'environnement. La codécision confere au $\mathrm{PE}$ de véritables pouvoirs de codécision et en fait le colégislateur, à côté du Conseil. Mais, c'est le traité d'Amsterdam qui fait disparaître la procédure de coopération au profit de la codécision. Pourtant, il faut remarquer que, à la différence des parlements nationaux, le PE n'a toujours pas l'initiative législative.

Le PE peut délibérer sur toutes les questions relatives à l'UE et émettre des avis. Les avis du PE ne lient pas les autres institutions.

Le PE peut poser des questions orales ou écrites auxquelles les autres institutions doivent répondre. 
Dans l'hypothèse ou il existe la consultation obligatoire, on est en présence d'un système de co-décision. Un acte qui n'est pas accompagné de l'avis conforme ${ }^{20}$ nécessaire est invalide comme entaché, non plus d'un vice de forme, mais d'incompétence.

Puis, il existe la seconde hypothèse - là où la consultation obligatoire est suivie par l'avis facultatif.

Le Parlement a la mission de contrôle. La Commission présente chaque année le rapport annuel ainsi que son programme de travail pour l'année à venir. Le Conseil rend le compte chaque année du développement de l'UE.

Le Parlement peut s'exprimer de sorte qu'il contraint la Commission à démissionner. Dans ce cas là, on parle d'une motion de censure ${ }^{21}$.

Le PE comprend 626 membres aujourd'hui. Le traité d'Amsterdam introduit sur proposition du PE lui-même la limite supérieure de 700 députés au PE.

Le Parlement peut intenter une action auprès delà CJCE. Il peut introduire un recours en carence contre d'autres institutions communautaires au cas où celles-ci omettraient de remplis leurs obligations conformément aux traités.

De leur côté les citoyens peuvent soumettre sous la forme de pétitions leurs demandes ou leurs plaintes à la commission des pétitions du PE. Le PE est également informé par le Médiateur européen.

Le PE veille sur la nomination du Président de la Commission ainsi que de la Commission dans son ensemble nécessite l'approbation du PE.

\subsection{Le Conseil de l'Union Européenne (Conseil des ministres)}

Avant qu'on se lance dans l'explication des problèmes du fond, il faut expliquer certains points qui peuvent causer la confusion. Le Conseil de l'UE ou le Conseil des ministres n'est pas le Conseil

20 L'avis conforme est exigé dans les domaines suivants : droits attachés à la citoyenneté, attribution de missions spécifiques à la BCE, modification des statuts de la BCE, organisation et fonctionnement des fonds à finalité structurelle, conclusion des accords d'association.

${ }^{21}$ La plus connue des motions de censure est celle du 1999 quand la Commission était obligée de donner sa démission le 15 mars. 
Européen ${ }^{22}$. Il s'agit de deux institutions différentes. En plus, il faut se méfier de toute ressemblance avec le Conseil d'Europe, qui reste hors l'UE, malgré le fait que c'est très fréquent de l'assimiler avec cette intégration européenne, d'autant plus que la route vers l'UE mène par le Conseil d'Europe ${ }^{23}$.

Le Conseil de l'Union européenne ${ }^{24}$ et le Conseil européen sont les institutions communautaires où se négocie l'équilibre entre les intérêts de chaque Etat membre et l'intérêt communautaire. Les intérêts particuliers des Etats membres sont défendus par les délégations gouvernementales des Etats membres.

Le Conseil se présentait dans le système institutionnel communautaire comme l'institution représentative des Etats membres. Il s'agit d'organe intergouvernemental dépositaire de la légitimité étatique. Ses compétences ont été étendues au-delà du domaine proprement communautaire et du fait du rôle dévolu au Conseil européen.

Le Conseil est régi dans sa composition et son fonctionnement en partie par les dispositions des traités et en partie par un règlement intérieur. Depuis 1993 le Conseil est dénommé "Conseil de l'Union Européenne ${ }^{25}$ et désigné comme tel dans tous les actes qu'il adopte, y compris dans le cadre de la politique étrangère et de sécurité commune et dans le cadre de la coopération dans les affaires intérieures.

La structure interne du Conseil se compose horizontalement d'un Conseil des affaires générales et de Conseils spécialisés, verticalement

${ }^{22}$ Le Conseil est subordonné politiquement au Conseil européen qui est composé des chefs d'Etat et de gouvernement ainsi que des ministres des affaires étrangères. Il est fréquent de poser la question concernant le Conseil européen et sa relation avec le Conseil. Peut-on le considérer comme une formation du Conseil ? Cette question n'est pas nouvelle. Certaines dispositions issues du tratié sur l'UE mentionnent le Conseil "réuni au niveau des chefs d'Etat ou de gouvernement " mais encore la pratique la plus récente montre, que le Conseil européen ne se borne pas à prendre des décisions de type politique, mais également de véritables décisions juridiques.

${ }^{23}$ Seulement le pays membre du Coseil de l'Europe peut devenir le candidat pour l'adhésion à l'UE.

${ }^{24}$ Avant l'entréc en vigueur du traité UE, la dénomination oficielle du Conseil était "Conseil des Communautés Européennes". Par décision du Conseil du 8 novembre 1993 cette dénomination a été remplacée par "Conseil de l'UE". On pourrait toutefois parler aussi des Conseils de l'UE car il existe actuellement 24 formations différentes du Conseil 
de trois niveaux de travail : du niveau ministériel ${ }^{26}$, du niveau des représentants permanents ${ }^{27}$ et du niveau des groupes de travail ${ }^{28}$.

Le Conseil est formé par un représentant de chaque Etat membre au niveau ministériel, habilité à engager le gouvernement de cet Etat. Par suite, seules les personnes ayant la qualité de membre du gouvernement d'un Etat pouvaient siéger au Conseil, à l'exclusion de toute autre et en particulier des fonctionnaires quel qu'en soit le rang. Ensuite, que la structure du Conseil pouvait se diversifier en formations suivant la correspondance entre, d'une part, les attributions propres de chaque membre des gouvernements nationaux et d'autre part les questions inscrites à l'ordre du jour. Enfin, ce texte n'en fondait pas moins l'unité de l'institution.

Le Conseil se réunisse dans des formations de composition et de compétence technique différentes.

Le Conseil a son siège à Bruxelles. Pendant le mois d'avril, de juin et d'octobre, le Conseil tient ses sessions à Luxembourg.

La présidence est assurée par chaque Etat membre pour une durée de six mois suivant un ordre fixé par les traités ${ }^{29}$. Selon une décision du Conseil européen de Bruxelles (décembre 1993) cette disposition devait être modifiée dans la perspective des nouvelles adhésions, l'ordre de rotation de la présidence, toujours semestriel, étant désormais fixé par

${ }^{25}$ Décision 93/591, J.O. n L 281 du 16 novembre 1993, p. 18

${ }^{26}$ Au niveau ministériel, le Conseil se réunit soit omme Conseil des affaires générales, soit comme Conseil ministériel spécialisé.

27 Les représentants permanents des Etats membres (les ambassadeurs acrédités auprès de l'UE constituent un comité représentant le niveau de travail intermédiaire du Conseil. Ce Conseil est appelé plus fréquemment Coreper. A l'origine, il se composait d'un seul représeentant permanent par Etat membre ainsi que d'un représentant de la Commission.

${ }^{28}$ Pour les délibérations et les négociations techniques proprement dites. Les groupes de travail sont composés de fonctionnaires spécialisés des ministères natioaux et la Commission y est représentée au même niveau. La plupart des groupes de travail sont institués pour une tâche très précise. Les groupes de travail ne peuvent ainsi délibérer que d'une matière clairement délimitée et sont dissous à l'issue de la procédure.

${ }^{29}$ A l'heure actuelle, Danemark préside. D'après l'agenda, il est prévu qu'en 2003 la

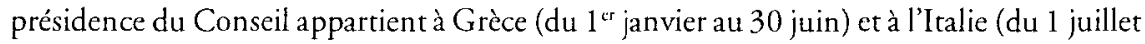
au 31 décembre) 
le Conseil statuant à l'unanimité. Il en va de même pour le Conseil européen dont la présidence est assurée de la même manière ${ }^{30}$.

Pour chaque session, le président établit un ordre du jour provisoire qui est adressé aux membres du Conseil et à la Commission au moins quatorze jours avant le début de la session.

Le pouvoir de décision ou le pouvoir d'arrêter les actes contraignants, était distribué par les traités entre la Commission et le Conseil. Or, depuis les révisions des traités constitutifs, on peut parler plutôt d'un triangle institutionnel ${ }^{31}$.

Pour la prise de décision du Conseil, le tratié CE prévoit en principe trois règles de vote: l'unanimité, la majorité qualifié et la majorité simple.

Dans la première hypothèse le traité exige l'unanimité, c'est-à-dire les voix de tous les membres du Conseil. La deuxième hèpothèse est celle des avis conformes. L'avis est réputé adopté si la proposition de la Commission recueille l'accord de la majorité absolue des représentants des Etats membres. Si, par contre, le traité n'impose ni l'unanimité ni une majorité qualifiée, les décisions sont risses à la majorité absolue des membres qui composent le Conseil.

Les voix des membres du Conseil sont affectées d'une pondération des voix. Cela signifie que les voix des Etats membres se voient affecter un coefficient de pondération. La limité minimum d'obtention de la majorité qualifiée est fixée à environ $71 \%$ du total des voix. Le nombre de voix suffisant pour empêcher d'atteindre la majorité qualifiée est appelé minorité de blocage. Le nombre des voix constituant la majorité qualifiée varie suivant qu'il s'agit pour le Conseil de statuer sur une proposition de la Commission ou dans les autres cas. Lorsque le Conseil statue sur proposition de la Commission les délibérations sont acquises si elles ont recueilli 62 voix.

30 La présidence a une grande importance pour le travail du Conseil. Lxercer les foncitons de la présidence signifie un grand prestige politique. Mais le préstige n'est pas la seule conséquence. L'Etat membre qui préside est chargé de fixer les séances, d'établir l'ordre de jour, ainsi que priorités politiques influence le rythme d'evolution de l'intégraion européenne, a cause du système de rotation, et par suite les problèmes prioritaires à resoudre changent pratiquement touts les six mois. Il est probable que ce système sera changé dans la perspective de l'élargissement.

${ }^{31}$ La Commission, le Conseil et le Parlement. 
Le Conseil ou les représentants des Etats membres désignent, entre autres, les membres de la Commission, de la CJCE, du TPI et de la CC.

Le Conseil est compétent pour conclure des tratiés internationaux au nom de la Communauté et il représente la Communauté vers l'extérieur.

Le Conseil ne peut s'écarter de la proposition de la Commission qu'en statuant à l'unanimité.

\subsection{Le Conseil Européen}

Le Conseil européen est né de la pratique des sommets des chefs d'Etat ou de gouvernement (Sommets européens). En 1974 il a été décidé que ce sommet se tiendrait régulièrement à l'avenir, sous l'appellation de Conseil européen. Par l'AU le Conseil européen est consacré pour la première fois dans les traités communautaires et dans le traite sur l'UE il se voit confier un ensemble de tâches plus clairement définies.

La tâche principale du Conseil européen est de donner les impulsions politiques nécessaires aux activités de l'Union ainsi que de fixer des orientations et objectifs politiques généraux. Il peut prendre certaines décisions.

Il peut exercer une certaine fonction de coordination, notamment à 1 'égard des diverses formations du Conseil de l'UE ou des politiques à poursuivre au sein des différents piliers de l'UE.

En ce qui concerne les décisions du Conseil Eruopéen, il faut remarquer que les procédures de vote ne font pas l'objet d'une réglementation claire. En règle générale, les documents, tels que déclarations et conclusions de la présidence, sont adoptés par consensus. Mais il existe aussi des cas, notamment lors de la convocation des conférences intergouvernementaux, où par analogie avec le Conseil de l'UE, les règles de vote de ce dernier ont été appliquées.

\subsection{La Commission Européenne}

Depuis $1967^{32}$, la Commission désigne l'institution des communautés qui, dans l'esprit des auteurs des traités institutifs, était destinée à représenter

\footnotetext{
${ }^{32}$ Auparavant, il existait la Haute Autorité, prévu par chacun des traités institutifs.
} 
chaque Communauté en tant que telle, à définir et à promouvoir l'intérêt commun, indépendamment des composantes étatiques de ces organisations. Le traité sur l'Union en a sensiblement modifié la situation en la plaçant dans une certaine dépendance du Parlement européen.

La composition de la Commission est fixée par les traités ${ }^{33}$. L'organisation de la Commission reproduit le modèle d'une structure de type gouvernemental comportant le collège, chacun de ses membres, les services et les organes auxiliaires.

La Commission possède un secrétariat général, placé sous l'autorité d'un secrétaire général, dont le rôle est très étendu.

La Commission dispose d'un ensemble de services qui forment une seule administration. Ces services sont structurés en directions générales et services assimilés, articulés à leur tour en directions, et celles-ci en unités. Elle peut constituer des structures temporaires, des groupes de travail et différents comités.

La Commission est composée de dix-sept membres choisis à raison de leur compétence générale et offrant toutes les garanties d'indépendance. Cet effectif peut être modifié par le Conseil statuant à l'unanimité, étant précisé que seuls les nationaux des Etats membres peuvent être membres de la Commission et que celle-ci doit comprendre au moins un national de chacun des Etats membres, sans que le nombre des membres ayant la nationalité d'un seul Etat soit supérieur à deux. Le Conseil européen de Bruxelles avait fixé à 21 cet effectif par addition de quatre nouveaux membres, un par nouvel Etat adhérent. La défection de la Norvège ramène cet effectif à vingt $(5 \times 2+10 \times 1)$.

Les membres de la Commission sont nommés pour un mandat de cinq ans renouvelable. Ils doivent exercer leur activité en toute indépendance, notamment vis-à-vis de leurs pays et de leurs gouvernements et sont tenus de veiller à l'intérêt général de la CE. Ils ne doivent ni solliciter ni accepter d'instructions des gouvernements ou d'autres organismes. Les Etats membres sont tenus de ne pas chercher à influencer les membres de la Commission ${ }^{34}$.

Indépendamment des décès, ce mandant pourra être écourté soit collectivement par l'adoption d'une motion de censure par le Parlement

\footnotetext{
${ }^{33}$ Art. $157^{\circ} \mathrm{CE}$, Art. 9० $\mathrm{CECA}$, Art. $12^{\circ} 6 \mathrm{CEEA}$

${ }^{34}$ Zu Martin, op.cit, p. 24
} 
européen soit individuellement à la suite d'une démission volontaire ou d'une démission d'office.

En cas de décès ou de démission du président, celui-ci sera remplacé pour la durée du mandat restant à courir, suivant la procédure générale.

Les fonctions des membres de la Commission sont évidemment incompatibles avec une autre activité, rémunérée ou non.

En cas de violation de ces obligations, la Cour de justice saisie par le Conseil ou par la Commission, peut, selon le cas, prononcer la démission d'office ou la déchéance du droit à pension de l'intéressé ou d'autres avantages en tenant lieu.

Dans la procédure législative, la Commission a un monopole d'initiative. "La Commission propose, le Conseil dispose ». Le mode normal d'expression de l'initiative est la proposition. Or ce n'est pas le seul mode. Il est possible que les demandes de la CJCE, deviennent le point de départ ou de déclenchement des procédures, qui parviennent à une recommandation. En plus, le Parlement européen, peut à la majorité des membres, demander à la Commission de soumettre toute proposition appropriée sur les questions qui lui paraissent nécessiter l'élaboration d'un acte communautaire pur la mise en oeuvre du traité. Ensuite, le Conseil et le Parlement peuvent inviter la Commission à leur soumettre une proposition d'acte juridique, mais ceci n'a aucun effet juridiquement obligatoire pour la Commission. Le but de cette construction est de laisser la Commission agir comme un filtre indépendant afin de ne pas remplir l'agenda politique communautaire avec une multiplicité d'initiatives n'obéissant qu'à la logique d'intérêts nationaux.

Dans l'exercice de ses compétences, la Commission est uniquement tenue de veiller à l'intérêt communautaire. Au sein du Conseil, elle est toujours représentée et à tous les niveaux de travail du Conseil. Elle n'y a pas le droit de vote, mais dispose du droit d'être entendeur.

Le rôle de la Commission en tant que représentante de l'intérêt communautaire est particulièrement important lors des décisions à la majorité au sein du Conseil : la conformité de l'acte juridique avec la proposition de la Commission garantit à la minorité au moins que l'intérêt communautaire est considéré comme respecté par la Commission.

La Commission veille au respect par les autres institutions et par les Etats membres de leurs obligations découlant du droit 
communautaire ${ }^{35}$. C'est pourquoi elle est appelée la gardienne des traités.

La Commission ne possède pas de pouvoirs d'exécution propres que dans le domaine de la concurrence, les aides d'Etat, et dans les domaines qui étaient couverts par le traité CECA.

Commission établit l'avant projet du budget qui est arrêté en dernier ressort par le Conseil et le Parlement. Elle est également chargée en grande partie de sa bonne exécution.

La Commission représente la Communauté auprès des Etats membres, des pays tiers et des organisations internationales. Elle possède ses propres représentations dans tous les Etats membres ainsi que des délégations dans ne centaine de pays dans le monde ainsi qu'auprès de nombreuses organisations internationales.

\section{Les institutions de contrôle}

Afin que l'intégration puisse devenir une réalité effective, des mécanismes visant à garantir l'application et l'interprétation uniforme du droit communautaire sont indispensables. Cette réflexion aboutit, dès la création de la CECA, à l'institution d'une Cour de justice, de structure supranationale et indépendante des Etats membres. De même, la coopération des différents organes communautaires ainsi que la répartition des compétences entre ceux-ci entre le niveau communautaire et les Etats membres ont laissé apparaître souhaitable la possibilité de recours à une instance judiciaire obligatoire. Les compétences correspondantes ont également été attribuées à la Cour de justice. Lors de la création de la CEE et de la CEEA, au lieu d'instituer des cours séparées, il a été décidé d'étendre les compétences de la Cour existante aux nouvelles Communautés. Certe cour est devenue ainsi la Cour de justice des communautés européennes, appelée également Cour européenne de justice ${ }^{36}$.

${ }^{35}$ La Commission peut introduire devant la CJCE une procédure en manquement d'Etat, recours en annulation ou en carence ou agir contre des violations du droit communautaire commises par le Conseil, le Parlement, l'Institut monétaire ou de la BCE. Dans certains domaines elle peut également agir contre des infractions commises par des personnes physiques et morales, par exemple dans le cadre du droit de la concurrence.

${ }^{36}$ Art. $220^{\circ}$ (ex 1640) TCE à $24^{\circ} 5$ (ex 188) ainsi que le protocole $\mathrm{N}^{\circ} 11$ (ex B) TCE portant statut de la CEJ 


\subsection{La Cour de Justice des Communautés Européennes}

La CJCE était prévu dans la structure originaire des institutions mais ce n'est pas le cas du Tribunal de première instance, prévu par l'Acte unique. La TPI a été effectivement instituće par une décision en $1988^{37}$.

La CJCE est composée de juges et d'avocats généraux. Elle dispose d'un greffier, assisté d'adjoints.

Au départ, la CJCE était formée de treize juges. Ce nombre était celui qui résultait en dernier lieu des actes d'adhésion de l'Espagne et du Portugal, illustrant la pratique suivant laquelle chaque adhésion entraîne une augmentation de l'effectif des juges, de manière à permettre aux nouveaux Etats membres d'y compter l'un de leurs ressortissants.

Les juges et les avocats généraux sont choisis parmi les personnalités offrant toutes garanties d'indépendance et qui ou bien réunissent les conditions requises pour l'exercice dans leur pays de plus hautes fonctions juridictionnelles ou bien sont des jurisconsultes ayant des compétences notoires. La nomination est faite d'un commun accord des gouvernements des Etats membres, de sorte que si juges et avocats généraux ont bien été présentés par leur Etat de nationalité, ils ne doivent pas à celui-ci une nomination, qui est collective.

Le mandat est de six ans, un renouvellement partiel intervenant tous les trois ans. Il existe la possibilité de renouvellement du mandat.

Les fonctions de juge et celles d'avocat général sont frappées d'un certain nombre d'incompatibilités destinées à garantir l'indépendance de leurs titulaires. Sont notamment interdits l'exercice de toute fonction publique ou administrative et celui de toute activité professionnelle, rémunérée ou non; sauf dans ce second cas, dérogation exceptionnelle qui, par exemple, peut concerner des fonctions d'enseignement.

A leur entrée en charge, juges et avocats généraux prêtent serment. Ils bénéficient d'une immunité de juridiction qui s'étende au-delà de la fin de leur mandant et que seule la Cour de justice peut lever. Ils ne peuvent être privés de leurs fonctions ou déchus des prérogatives qui y sont attachées, que par un jugement unanime de la Cour, délibérant hors la présence de l'intéressé.

${ }^{37}$ Décision 88/591 du Conseil du 24 octobre 1988. JO n L 319 du 25 novembre 1988 
La cour fixe son statut.

Il existe pour l'essentiel six types de procédure devant la CJCE. Ce sont la procédure en manquement, le recours en annulation, le recours en carence, l'action en réparation, la procédure de renvoi préjudiciel et l'avis.

Par rapport à l'UE prise dans son ensemble, la CJCE n'était compétente après l'entrée en vigueur du traité de Maastricht que pour le pilier communautaire et les dispositions finales.

Le traité d'Amsterdam a considérablement élargi les compétences de la CJCE, notamment afin de renforcer la protection des droits de l'homme et des libertés fondamentales dans l'UE, ainsi qu'aux certaines questions relevant du troisième pillier de l'UE. Ceux-ci liaient l'Union déjà auparavant, vu que l'Art. $6^{\circ}$ (ex F) TUE faisait explicitement référence à la Convention européenne des droits de l'homme. Par le traité d'Amsterdam, l'Art. 46० (ex Art. L) a été amendé afin de soumettre le respect des droits de l'homme et des libertés fondamentales par les organes de l'UE à la jurisprudence de la CJCE. En outre, les nouvelles dispositions sur la coopération renforcée sont soumises au contrôle de la CJCE.

\subsection{Le Tribunal de Première Instance}

Comme mesure d'allégement du travail de la CJCE il lui a été adjoint en 1988 le Tribunal qui statue en première instance pour certains types de litiges.

Le TPI est saisi des recours formés dans le cadre du droit de la fonction publique, du droit de la concurrence, dans le domaine du charbon et de l'acier ainsi que pour tous les recours directs des personnes physiques et morales contre une institution communautaire (à l'exception des recours formés dans le cadre du droit antidumping). Un arrêt du TPI peut faire l'objet d'un recours auprès CJCE.

\subsection{La Cour des Comptes}

La Cour des comptes figure parmi les institutions dans l'énumération que donnent les traités des communautés européennes depuis le traité sur l'Union. 
La Cour des comptes a été crée par le traité du 22 juillet 1975. Sa reconnaissance a été destinée à renforcer l'autorité.

La Cour des comptes est chargée du contrôle des comptes. Ce n'est pas une institution juridictionnelle.

Elle a son siège à Luxembourg.

Les membres de la Cour ne peuvent pendant la durée de leurs fonctions exercer d'autre activité professionnelle, rémunérée ou non.

Les membres de la Cour ne peuvent être relevés de leurs fonctions, ni déclarés déchus de leurs droits à pension ou d'autres avantages en tenant lieu, que si la Cour de justice constate, à la demande de la Cour des comptes, qu'ils ont cessé de répondre aux conditions requises ou de satisfaire aux obligations résultant de leur charge. La démission volontaire ou d'office sont possibles.

La Cour des comptes a pour mission d'examiner la légalité et la régularité des recettes et des dépenses de la Communauté ainsi que de faire respecter les critères d'une bonne gestion financière. La Cour des comptes décide en toute liberté des domaines qu'elle soumet à un contrôle plus détaillé.

La Cour des comptes peut contrôler les organismes relevant des Etats membres, et des entreprises et des personnes privées dans la mesure où il s'agit de vérifier l'utilisation conforme au droit communautaire des subventions de la CE. Ce travail, elle fait en collaboration avec les autorités de contrôle nationales.

La Cour des comptes est autorisée à adopter des prises de positions dans le cadre de certaines procédures de décision. C'est le cas, par exemple, s'agissant des propositions de changements de règlements financiers du budget communautaire ou de propositions visant la lutte contre la fraude.

\section{La nécessite de la reforme des institutions de l'UE}

L 'élargissement de l'Union européenne et l'adhésion des nouveaux Etats posent de nouveaux problèmes. La nécessite de la reforme est évidente, vu que la structure existante a déjà montré certains défauts dans la pratique. Si elles ne peuvent pas gérer les intérêts des 15 Etats 
membres à l'heure actuelle, il est difficile à imaginer que demain elles pourront le faire pour les 25 ( 27 ou 28 ).

Les premières idées de la reforme voient le jour déjà lors les travaux préparatifs de la conférence intergouvernementale (CIG) pour le traité d'Amsterdam. La conclusion à cette époque là est qu'il faut renforcer les institutions. La reforme institutionnelle sous-entend le changement de la procédure législative. Il faut multiplier les cas de prise de décision à la majorité et diminuer ceux à l'unanimité. Ainsi on renforcera le rôle du Parlement. Pourtant, il est jugé que le monopole d'initiative sera très difficile à transférer de la Commission au Parlement.

Les institutions législatives doivent changer de la taille. En même temps il faut limiter le nombre des commissaires dans la Commission, la pondération des voix au sein de Conseil et le nombre des députes au Parlement. C'est un sujet difficile à gérer si les Etats membres ne veulent pas renoncer aux priorités nationales. On est les témoins d'une nouvelle phase d'évolution de l'UE. L'affirmation du rôle des nations au sein des structures communautaires mène à la confédération. Les travaux sur la Convention continuent, et doivent aboutir à l'accord lors la prochaine CIG, prévu pour 2004.

Lors le dernier sommet de Conseil européen à Séville le 21 et 22 juin 2002, l'accord concernant la reforme du Conseil a vu le jour. En effet, on voit des changements multiples, concernant le Conseil des ministres et le Conseil européen. Pourtant, il reste l'impression d'une reforme plutôt technique, car il n'y a pas d'accord sur les questions de fond, tel que les compétences, la pondération des voix, le representement des Etats, les décisions à prendre à la majorité qualifiée au lieu de l'unanimité.

Cette année s'est montrée difficile. Plusieurs reformes et plusieurs réunions importants était sur l'agenda de l'UE. Mais les élections en Allemagne ont ralenti le processus de négociations. Les prochains mois donneront la réponse aux certains questions. Mais, pour la reforme institutionnelle il faut attendre encore un peu.

L'élargissement de l'Union et l'adhésion des premiers Etats est prévu pour l'année 2004. Avant cette date les institutions européennes doivent être prêtes pour l'Europe de 25. 